\title{
LA SALUD MENTAL EN LOS PORTUGUESES DE ENSEÑANZA SUPERIOR FRENTE A LA TRANSICIÓN AL MERCADO DE TRABAJO
}

\author{
Ana Luísa Cordeiro Vicente \\ Psicóloga en la CERCIAG; Doctora por la Universidad da Extremadura \\ aluisavicente@sapo.pt
}

Fecha de Recepción: 24 Enero 2019

Fecha de Admisión: 30 Abril 2019

\section{RESUMEN}

En el actual entorno de crisis económica y del agravamiento del desempleo, la preocupación es, sobre todo, con el deterioro de la salud mental de los estudiantes universitarios que saldrán al mercado laboral, ya que esta transición plantea problemas a nivel de la salud mental de los estudiantes universitarios, contribuyendo al desarrollo de niveles más elevados de ansiedad y de estrés.

Este artículo es una síntesis de un estudio de investigación que trata de determinar la existencia de una relación entre la salud mental en los estudiantes de educación superior portugués debido a la transición al mercado laboral. Mirando corroborar este fin, se utilizó una muestra de 302 sujetos de diferentes instituciones de educación superior en Portugal. Para la evaluación de las variables del estudio se utilizaron la versión en portugués del Breve Inventario de Síntomas (BSI) (Canavarro, 1999), la versión en portugués del Inventario de Salud Mental (MHI) (Ribeiro, 2001), y la Escala de Desánimo frente al empleo (adaptado de Job Hopelessness Scale - JHS).

Los principales resultados muestran que existe una asociación entre el desánimo frente a la demanda de empleo y la sintomatología psicopatológica evaluada por el BSI, y que existe una asociación entre el desánimo frente a la demanda de empleo y el distress y el bienestar psicológico evaluado por el MHI.

Palabras clave: salud mental; distress; desaliento; mercado de trabajo; estudiantes universitarios

\footnotetext{
ABSTRACT

Mental health in the portuguese of higher education in front of the transition to the labor market. In the current environment of economic crisis, and rising unemployment the concern is primarily with the deterioration of Mental Health of university students who will go to the labor market, since this transition poses highlighted problems with the mental health of university students, contributing to the development of higher levels of anxiety and stress.
} 
This article is a comprehensive summary of a research, who sought to determine whether there is a relationship between mental health in higher Portuguese education students face the transition to the labor market. Looking corroborate this end, we used a sample of 302 students, of different establishments of Higher Education of Portugal. For the assessment of study variables were used the portuguese version of the Brief Symptom Inventory (BSI) (Canavarro, 1999), the portuguese version of the Mental Health Inventory (MHI) (Ribeiro, 2001), and the Job Hopelessness Scale (JHS).

The main results show that there is an association between the discouragement towards job search and the psychopathological symptomatology evaluated by the BSI, and that there is an association between the discouragement towards the job search and the distress and the psychological well-being evaluated by the MHI.

Keywords: mental health; distress; discouragement; labor market; university student

\section{INTRODUCCIÓN}

El pleno empleo es una utopía? Es una ilusión esperar el regreso de una fuerte economía, regular y competitiva? 0, por el contrario, debemos resignarnos a una perspectiva de exclusión económica y social en los próximos tiempos. Claro que no. El futuro económico, también está en las manos de los futuros profesionales que todos los años egresan de las Universidades Portuguesas. El crecimiento y el pleno empleo deben ser para Portugal y para Europa un objetivo a corto y a medio plazo.

La búsqueda de empleo y el desempleo son acontecimientos de vida inductores de stress por las mudanzas que traen para la vida de los individuos. Estas condiciones no tienen un impacto psicológico igual para todas las personas, pues existen variados factores que se deben tener consideración a lo largo de este proceso, que nos pueden ayudar a prever la sintomatología y el grado de stress que un estudiante podrá venir a sentir en esta situación.

La transición para la Universidad coloca en destaque los problemas de los estudiantes, contribuyendo para el desenvolvimiento de los niveles más elevados de ansiedad y de stress. Los problemas que los estudiantes presentan son variados en esta fase de su vida, (Pereira, 1997 cit. in Ferraz \& Pereira, 2002, p.150): en primer lugar son los problemas personales como el homesikness, la soledad, la timidez, limitaciones en las competencias sociales y en la toma de decisiones, la sexualidad, las perturbaciones emocionales. En segundo lugar son los problemas académicos, como las dificultades de relacionamiento con los profesores y compañeros, competencias de estudio, rendimiento escolar, ansiedad y el stress en situación de evaluación, etc. En tercer lugar son los problemas financieros y de gestión de la casa con mayor énfasis en la acomodación y hábitos alimenticios y los problemas relacionados con la seguridad. En cuarto lugar son los niveles de ansiedad, depresión, de la baja auto-estima a la fase de la búsqueda de un empleo y la inserción en el mercado de trabajo. La búsqueda de un empleo y su posible dificultad en conseguirlo puede promover la diminución de la auto-estima, y la alteración en la vida psíquica, ocurriendo una desestructuración con serias repercusiones en la salud mental de los individuos. Los autores Revuelta et al. (2016 cit. in Medina-Mesa, Martínez-García, Morales-Sánchez, \& Gil-Olarte, 2018, p.210) define la salud mental como "el estado de equilibrio o estabilidad de una persona con su contexto socio-cultural. Implica un bienestar emocional, psíquico y social relacionado directamente con la forma en la que pensamos, sentimos y tomamos decisiones".

Las incertezas sobre la economía actual y como consecuencia de la crisis social, las tazas de desempleo son una realidad. El problema del desempleo es extremamente complejo, empezando por las explicaciones para el surgimiento de este fenómeno. La evaluación organizacional debe tener en cuenta las características individuales de cada uno de los individuos, la formación profesional, la 
dislocación geográfica, la mudanza de profesión, la continuación de los estudios o la ausencia de ellos. Además de la posibilidad de acceso a una formación científica y profesional especializada, a frecuencia de la Enseñanza Superior constituye también, para una gran parte de los estudiantes, una oportunidad de promoción de sus competencias transversales, tanto a nivel comportamiento y de actitudes, como el nivel personal y social (Seco, Filipe, Pereira, \& Alves, 2009). Las personas siempre esperan que, estudiando más tiempo, tendrán una garantía de empleo y, luego, después de los 16017 años de estudio, de forma a obtener una licenciatura, tendrán una mayor oportunidad de desenvolver una carrera estable, con la posibilidad de progresión, un sueldo justo, buenas condiciones de trabajo y realización profesional (Castro \& Pego, 2000; Cruz, 2009). Sin embargo, en los días de hoy, la construcción de una Carrera Profesional por parte de los jóvenes licenciados es muy dificultada, principalmente por la existencia de un mercado inestable, con mudanzas tecnológicas, reestructuraciones empresariales y precariedad, mas sobretodo pelos elevados índices de desempleo, que afectan particularmente a los jóvenes a la salida del sistema educativo. También en este proceso de inserción laboral de los jóvenes y por tanto de su emancipación, provoca la búsqueda de nuevas alternativas de empleabilidad, que le permitan alcanzar el grado de autonomía personal y económica necesarias para conseguir el proceso de avance social y de bienestar (López, 2012 cit. in Ballester \& Fernández, 2015, p.172).

\section{FINALIDAD Y OBJETIVOS DEL ESTUDIO}

La escogencia de este tema está relacionado con el hecho de el desempleo y sus consecuencias en la salud mental, hayan a lo largo de los años, poco espacio en el debate de la Psicología Clínica, sin embargo, la manifestación cada vez más intensa y frecuente de los fenómenos psicológicos que advienen del desempleo (depresión, bajo auto-estima, relaciones familiares degradadas, entre otras), sugiere la necesidad de una actuación directa de esta ciência.

El presente artículo describe las características y analiza las relaciones entre salud mental y las expectativas de los estudiantes en pro de conseguir o no un fututo empleo. El objetivo general de esta investigación es verificar la relación entre las variables (sexo, edad, nivel de escolaridad, residencia habitacional, y la situación de acuerdo al trabajo) y la sintomatología psicopatológica, la angustia y el bienestar psicológico en los estudiantes de la Enseñanza Superior, y la relación de estos de acuerdo a las expectativas individuales en lo que respecta a conseguir o no un futuro empleo: analizar el estado de salud mental en los estudiantes portugueses de la enseñanza superior de acuerdo a la transición para el mercado de trabajo.

De manera a corresponder con lo que delimitamos, desdoblamos este objetivo general en dos objetivos específicos que esquematizan, aquello que exigimos como meta de trabajo de investigación y que, en simultáneo, también servirán de orientación para la direccionalidad de este trabajo. Así que, de seguida destacamos los objetivos específicos, para la muestra seleccionada: 1) Identificar y verificar las variaciones relacionadas con el sexo, la edad, el nivel de escolaridad y situación en relación al trabajo, su sintomatología psicopatológica, en la angustia y en el bienestar psicológico en los estudiantes universitários; 2) Estudiar la relación existente entre la salud mental y los síntomas psicopatológicos como el desánimo en base a la transición para el Mercado de Trabajo.

Partimos de la idea que la salud mental de los estudiantes de la Enseñanza Superior está influenciada por un conjunto de factores relativos a sus características socio demográficos, a las características del área de formación superior que frecuentan, así como las asociadas a las características del mercado de trabajo/economía actual. Estos supuestos, fundamentados con la literatura consultada y las convicciones relacionadas con los trayectos que nos trazamos profesionalmente, orientaron la formulación de las suposiciones que seguidamente se exhiben: Hipótesis 1: Existen diferen- 
cias del sexo y de la edad de los sujetos relativamente a la Salud Mental; Hipótesis 2: Los sujetos con la habilitación de Máster presentan mejor salud mental en relación a los que poseen sólo Licenciatura; Hipótesis 3: Los sujetos que viven lejos de su domicilio habitacional presentan un bajo índice de salud mental; Hipótesis 4: Los individuos que trabajan presentan un bajo índice de angustia, por lo que evidencian una mejor salud mental; Hipótesis 5: Los individuos con licenciatura presentan un mayor desánimo en relación a la búsqueda de empleo y un bajo índice de salud mental; Hipótesis 6: El desánimo de acuerdo a la búsqueda de empleo siendo inversamente proporcional al índice de los síntomas psicopatológicos; Hipótesis 7: El desánimo por la búsqueda de empleo influenciada por la angustia y el bienestar psicológico.

\section{METODOLOGÍA E INSTRUMENTOS DE ACCESO A LOS DATOS}

Siguiendo una metodología de investigación cuantitativa, el presente estudio obedece a un diseño no experimental, escogido para el estudio descriptivo o de observación correlacionado, visto que no existe una manipulación de variables independientes ni estabelecimiento de causalidad, pero sin la descripción de los fenómenos y análisis de las relaciones entre las variables en estudio (Ribeiro, 1999).

El universo que se consideró fue de dos Universidades Portuguesas (Universidad de Coimbra y la Universidad de Algarve) y tres Institutos Superiores (Instituto de Estudios Superiores de Fafe, Escuela Superior de Educación de Coimbra y la Escuela Superior de Educación Almeida Garrett), siendo el universo para la totalidad del presente estudio. De este universo, seleccionamos cinco, teniendo en cuenta la distribución geográfica de los mismos, denominadamente su localización Norte/Sur, procurando fundamentalmente un equilibrio que refleja la realidad nacional. La muestra de la población está constituida por 302 participantes. La búsqueda de datos fue efectuada por la investigadora en los referidos estabelecimientos de Enseñanza Superior, y los datos fueron recogidos teniendo como único destino la construcción de la base de datos para esta investigación.

Fue utilizado un conjunto de cuatro instrumentos estandarizados para la pesquisa de datos. Para la descripción de las principales características de la muestra fue utilizado un "Cuestionario Socio demográfico" al estudiante. La evaluación de la salud mental fue efectuada con base a dos instrumentos distintos: las versiones portuguesas de Mental Health Inventory (MHI) (Ribeiro, 2001) y de Brief Symptom Inventory (BSI) (Canavarro, 1995, 1999). El primero fue seleccionado por demostrar una dimensión positiva (bienestar psicológico, estado de salud mental positivo) y otra negativa (destres psicológico, estado de salud mental negativo). La selección del segundo instrumento se debe al hecho que caracteriza la principal sintomatología (El individuo clasifica el grado de cada problema o síntoma presentado y como afectó durante la última semana). La evaluación para medir las expectativas en lo que respecta a conseguir o no un empleo en el futuro, fue efectuada a través de Job Hopelessness Scale (JHS).

\section{PRESENTACIÓN Y DISCUSIÓN DE LOS RESULTADOS}

Después de haber obtenido los datos, se procedió al análisis estadístico de los mismos, con el auxilio del programa informático SPSS, versión 19.0. Los procedimientos estadísticos son realizados de acuerdo con los objetivos y supuestos, así como el tipo de variables en causa. Los dominios estadísticos serán estrictamente los descritos, su correlación e inferencia.

A groso modo, al identificar las características socio demográficas de nuestra muestra, podemos destacar la no existencia de una diferencia significativa entre el número de sujetos masculinos $(n=146)$ y femeninos $(n=156)$. La mayoría de los sujetos se encuentran solteros $(78,1 \%)$, con edades comprendidas entre los 20 y los 23 años y continúan residiendo con la familia parental $(82,5 \%)$. Por lo general, los sujetos entraron en la $1^{a}$ candidatura a la Universidad y en la $1^{a}$ opción del curso 
pretendido. Se encuentran frecuentando el último año de su respectiva Licenciatura $(85,8 \%)$ en comparación a los que frecuentan el Máster (13,9\%). Relativamente al trabajo, los sujetos en su mayoría se encuentran apenas estudiando $(64,6 \%)$, y cuando terminen su curso, pretenden trabajar en el área de especialización. La mayoría de los sujetos de la muestra se encuentran frecuentando el Curso de Educación Física-Deporto (26,5\%), seguidos del curso de Educación Básica (13,9\%) y del curso Educación Social (10,3\%). Verificándose que los restantes cursos son menos representativos. Después de la obtención de los datos, se procedió al análisis estadístico de los mismos. Las cuestiones de la investigación fueron sucintamente así analizadas:

\section{Hipótesis 1: Existen diferencias del sexo y de la edad de los sujetos relativamente a la Salud Mental}

Las diferencias de género poseen una importancia al nivel de la salud mental, principalmente en lo que concierne a algunas perturbaciones mentales y de comportamiento. Para responder a esta cuestión fueron analizadas las respuestas de los individuos relativamente a las dimensiones e índices de la escala de la muestra por sexo, y fueron analizadas las respuestas de los individuos al cuestionario $\mathrm{MHI}$ y BSI. Nuestros resultados indican que no existen diferencias de sexo relativamente a la salud mental. Tanto las mujeres como los hombres presentan puntuaciones semejantes en la angustia psicológica y en los indicadores evaluados de la sintomatología primaria. En relación a la evaluación de la salud mental por el MHI, ambos sexos presentan valores semejantes en todas las dimensiones negativas (ansiedad, depresión, pérdida del control emocional/comportamiento) y en las dimensiones positivas (afecto positivo y lazos emocionales), bien como en la angustia psicológica, en el bienestar psicológico y en índice global de la salud mental. En las escalas de la sintomatología primaria e índices del BSI, tanto las mujeres como los hombres revelaron medias semejantes en todas las dimensiones (somáticas, obsesiones-compulsiones, sensibilidad interpersonal, depresión, ansiedad, hostilidad, ansiedad fóbica, ideas obsesivas y psicopáticas), bien como en el índice general de los síntomas y en el índice de los síntomas positivos. Estos resultados van en sentido contrario a la posición del autor Canetto (1997 cit. in Rabasquinho \& Pereira, 2007, p.439) cuando refiere que las mujeres son más socializadas en el sentido de internalizar la angustia que los hombres, contribuyendo para los desordenes asociados a la depresión, ansiedad e ideas suicidas. Sin embargo, también este autor, concluye que estas diferencias pueden ser previsibles por la socialización, pudiendo ser mediadas más por los papeles del género estereotipados, que por las diferencias de comportamiento delante de los factores estresantes presentados por los géneros (Rabasquinho \& Pereira, 2007, p.439).

Con todo esto, los estudios realizados por Marks y Seiber (Cleary, 1987, Weissman, 1988, Robins, 1991 cit. in Rabasquinho \& Pereira, 2007, p.440) van a la misma dirección de nuestros resultados, o sea, en el sentido de alterar la concepción de que las mujeres presentan tasas más elevadas que los hombres de enfermedades mentales, contrariando la idea de que las mujeres presentan mayores dificultades en desempeñar diversos papeles de género comparativamente a los hombres, justificando la diferencia de sus resultados, delante de los de una gran mayoría de autores por el hecho de, que en esos estudios usasen escalas de selección, que incluyesen ítems relacionados con la depresión, ansiedad, síntomas somáticos, la angustia, síntomas específicos que son presentadas con alta incidencia en las mujeres.

Relativamente a la asociación entre la edad de los sujetos y la salud mental, los resultados muestran que no existen diferencias significativas, cuando fueron evaluados por el MHI en sus diferentes dimensiones e índices. La asociación de la edad con el MHI muestra todavía, que relativamente al índice de angustia psicológica y al índice de bienestar psicológico y al índice Global, estos 
presentan una asociación débil y negativa, lo que indica que cuanto menor sea la edad, mayor es el nivel de angustia y de bienestar psicológico. La misma tendencia es también verificada en los resultados del BSI, en sus dominios e índices para la edad, indicando para los dominios una asociación débil y positiva. 0 sea, en el Índice General de los Síntomas y en el Índice de los Síntomas Positivos podemos indicar una relación débil y positiva, lo que indica que cuanto mayor es la edad, mayor es el nivel de los síntomas positivos presentados.

Los resultados del presente estudio van en dirección a la posición de la teoría de Tartakovsky (2008) que refiere que la edad media de inicio para problemas de salud mental es la del grupo etario típico de la universidad, desde los 18 a los 24 años. También Wong et al. (2006) menciona que a pesar que la población en general, en la transición de la adolescencia para la edad adulta representa un período de elevado riesgo de depresión, los jóvenes que ingresan en la enseñanza superior pueden enfrentar nuevas mudanzas sociales e intelectuales que podrán contribuir para un aumento del riesgo de sufrir de depresión, ansiedade y stress (Pereira, 2009). 0 sea, los alumnos que presentan una menor edad, muestran un mayor nivel de Ansiedad, Depresión y Pérdida del Control Emocional/Comportamiento. Segundo Arnett (2006), el período de inicio de la edad adulta, transcurre entre los 18 y los 25 años de edad, ocurriendo en esta fase, importantes mudanzas cognitivas, de comportamiento y de personalidad, las cuales reflejan situaciones de ámbito personal, social y cultural, evidenciadas por el individuo (Porta-Nova, 2009).

Hipótesis 2: Los sujetos con la habilitación de Máster presentan mejor salud mental en comparación con los de la Licenciatura

Algunos estudios refieren que el menor nivel de habilitación influye negativamente o provoque un probable sufrimiento psicológico. Sin embargo, los resultados obtenidos en nuestro estudio no son definitivos para los dos niveles de habilitación, principalmente por el hecho de que ambas muestras presentasen tamaños muy diferenciados, o sea, los individuos en el último año de la Licenciatura ( $n=259)$, Máster $(n=42)$ y Doctoramiento $(n=1)$.

Para responder a esta cuestión fueron analizadas las respuestas de los individuos al cuestionario del $\mathrm{MHI}$ y del BSI. Los resultados obtenidos, refieren que no existen diferencias significativas entre la habilitación de la Licenciatura y la habilitación del Máster, y en relación a las dimensiones y a los índices del MHI. 0 sea, no existen diferencias significativas en cuanto al nivel de Bienestar psicológico y en el índice Global de la salud mental. Sin embargo, se verificó una pequeña diferencia al nível del índice de angustia psicológica para la Licenciatura y para el Máster, lo que indica que los sujetos que se encuentran a frecuentar la Licenciatura, presentan un mayor nivel de angustia psicológica. En lo que concierne a la relación entre la habilitación y a las escalas de sintomatología primaria e índices del BSI, los valores presentan algunas diferencias estadísticas (no muy relevantes), que presuponen que cuanto mayor es la habilitación (Máster), mayor es el nivel de síntomas positivos presentados. Estos resultados van al encuentro de los diferentes autores, Castro y Pego (2000 cit. in Cruz, 2009, p.24) que refieren que los licenciados desenvuelven expectativas vocacionales más elevadas y, por eso que cuando se hallan en una situación de no integración profesional, estos son el eje de grandes impactos psicosociales, pues viven en un escenario social indeleblemente marcado por la continua aceleración de la velocidad de mudanza en el paisaje profesional, en el cual la persistente inestabilidad profesional generando en los jóvenes una amarga sensación de no saber lo que hacer con tanta escolaridad y/o cualificaciones disponibles.

La educación se volvió para los portugueses, tal como para los países desarrollados de los continentes europeo y americano, en un elemento que permitió el ascenso social y la construcción de una sociedad con mayores niveles de igualdad social y económica. En nuestros días, la construc- 
ción de una carrera profesional por parte de los jóvenes licenciados está muy dificultada, principalmente por la existencia de un mercado inestable, con mudanzas tecnológicas, reestructuraciones empresariales y precariedad, pero sobretodo por los elevados índices de desempleo en Portugal, que afectan particularmente a los jóvenes al egresar del sistema educativo, proporcionando muchas veces la migración para otros países. De esta forma, la valorización de la Enseñanza Superior es una realidad en el mundo actual, y por esta razón, cada vez más, surge la tendencia en los licenciados un investimento en el proseguimiento de sus estudios, denominadamente al nivel de las pós-graduaciones, másteres y/o doctoramientos.

Hipótesis 3: Los sujetos que habitan fuera de su residencia habitacional presentan menor índice de salud mental

Fuimos analizar la existencia de las diferencias en la salud mental de los sujetos de acuerdo al hábitat y con su agregado familiar. En este contexto, en lo que concierne a la salud mental, diversos estudios han sido valorizados, pues permiten una mayor comprensión de la prevalencia de los trastornos mentales y de sus implicaciones para el individuo, incluyendo principalmente el apoyo/soporte familiar (Bener \& Tewik, 2006 cit. in Souza, Daher, \& Baptista, 2010, p. 145).

Para responder a esta cuestión fueron analizadas las respuestas de los individuos del cuestionario MHI y del BSI. Debido al hecho que nuestra muestra presentar valores muy contrarios, se consideró importante, realizar el estudio sobre las dos muestras más consistentes, o sea, la población que "No sale de casa" ( $n=249)$ y quien habita en un "Apartamento con otros estudiantes" $(n=30)$. A través de los resultados obtenidos podemos decir que no existen diferencias significativas entre estas dos poblaciones relativamente a las dimensiones y a los índices del MHI, con excepción de las dimensiones Afecto Positivo y Lazos Emocionales, que presentan para la residencia habitacional "No sale de casa" y para la residencia habitacional "Piso con otros estudiantes" respectivamente. Para las escalas de sintomatología primaria e índices del BSI, los valores no presentan diferencias estadísticamente significativas para la residencia habitacional, con excepción de la dimensión de Ansiedad Fóbica, que presenta con residencia habitacional "No sale de casa" y para los de residencia habitacional "Piso con otros estudiantes". Esta constatación apenas nos permite reflexionar con el estudio bibliográfico realizado, que nos refiere la existencia de una red de soporte social eficiente (en este caso los padres, hermanos, abuelos, etc.) permitiendo al individuo presentar un mayor índice de salud mental. De acuerdo con Sluzki (1996 cit. in Sousa \& Alarcão, 2007), la red de soporte social, en cuanto al nicho interpersonal del sujeto, se revela como base de apoyo fundamental, no sólo por el soporte funcional que proporciona, pero, similarmente, por el encuadramiento emocional y por el propio crecimiento que posibilita, contribuyendo así para la capacidad adaptativa del sujeto en situación de crisis, así como para el mantenimiento de su salud física y mental.

En este contexto, los estudiantes que viven en "un piso con otros estudiantes", reciben el apoyo de otros compañeros con quien residen, apoyo/fuente de soporte, puesto que su entrada en la universidad es, en la mayoría de las veces la primera vez en que el adolescente sale de su casa, frente al dilema de la separación de sus padres y de su familia. La transición de la Universidad para el mercado de trabajo, produce algunos problemas para los alumnos, contribuyendo al desarrollo de altos niveles de ansiedad y estrés por la posibilidad de no conseguir trabajo, por eso el soporte social es fundamental en esta etapa, sea de la familia, sea entre pares. Murgatroyd (1998 cit. in Costa, 2004) refiere que las relaciones humanas y el apoyo social tienen un efecto positivo para el control y prevención del estrés. 
Hipótesis 4: Los individuos qué trabajan tienen menor índice de angustia, luego tendrán mejor salud mental.

Para responder a esta hipótesis, se ha considerado significativo una comparación entre las dos muestras más grandes de la población, es decir, entre individuos que "solo estudian" (64,6\%) y los que "trabajan a tiempo completo" $(21,2 \%)$. Los resultados obtenidos, con respecto a los índices de angustia psicológica del índice global del MHI y otras dimensiones, no se observaron diferencias estadísticamente significativas. Relativamente a las escalas de síntomas primarios e índices del BSI, Ios valores representan algunas diferencias estadísticas (no muy significativas) para quien "solo estudia" y para quien trabaja "a tiempo completo". Estas diferencias, presuponen que quien trabaja, tiene niveles más altos de síntomas positivos. Relativamente al índice de angustia psicológica y al índice global del MHI, todos ellos apuntaron la ausencia de diferencias significativas entre los momentos en comparación, así como para las restantes dimensiones. Hemos verificado que por las escalas de sintomatología primaria y los índices del BSI, los valores exhiben algunas diferencias estadísticas (no demasiado significativas) sea por la situación en materia de empleo "solo estudia", sea para quien trabaja "a tiempo completo". Estas diferencias, presuponen que los que trabajan, tienen niveles más altos de síntomas positivos.

Como han referido Cardoso y Sampaio (1994, p.3), existen básicamente tres situaciones: i) el joven que sólo estudia, ii) el que estudia y trabaja y iii) el joven que estudia y necesita trabajar como imperativo de supervivencia, como ocurre en nuestro estudio. Estas situaciones "presentan diversas caras psicológicas y conductuales llevando para el campus universitario problemas también diversificados, urgentes y de relevancia incuestionable". Aunque estos autores traten de superar la dicotomía entre trabajo y estudio. Mostrando que la reconciliación de estas dosactividades pueden ser factibles, la concepción del trabajo, en realidad, aparece subordinada al proceso estudio - aprendizaje. Es decir el trabajo, sólo es deseable si integrado en la dinámica del proceso educativo, como parte del esfuerzo de "integración entre conocimiento y acción, teoría y práctica, información y formación, democratización y eficacia, desarrollo económico-social y madurez individual, diálogo y metodología didáctica".

Actualmente el desempleo se ha convertido en un problema discutido en todo el mundo debido al gran número de desempleados y a los problemas derivados de esa situación, los cuales se reflejan en la vida de cada individuo, pudiéndose centrar en la salud mental de esas personas (Barros \& Oliveira, 2009). Los autores Clarke y Critcher (1985, Glyptis, 1989 cit. in Cruz, 2009) afirman que existen cinco necesidades que se satisfacen mediante el empleo: la estructuración de tempo, en la que se establece un cierto grado de organización en el tiempo de las personas; el intercambio de experiencias, fuera del contexto familiar, o sea, la posibilidad de construir amistades y conocimiento con otras personas que no hacen parte de la familia; la unión de individuos alrededor de objetivos más allá de sí proprio, o sea, la colaboración con otros para alcanzar los propósitos que no alcanzarían solos, obtener estatus, identidad y por último, el fortalecimiento de su actividad, es decir, sentir que lo que se hace "merezca la pena" y contribuir hacia algo mejor. Así que, la satisfacción de tener un empleo permite a los individuos, la presentación de una mejor salud mental, por lo tanto, un menor índice de angustia y malestar. Segundo, Vlezina, Derriennic y Monfort (2004 cit. in Cruz, 2009), los desempleados, en este caso, que no trabajan, sólo estudian presentan niveles de autoestima baja y depresión que las personas que están empleadas. Sin embargo, nuestra muestra no presenta diferencias estadísticamente significativas en relación con la angustia psicológica, ya que la mayoría esta a terminar la graduación esperando empezar su función profesional después de su finalización.

Los estudiantes universitarios asumen así, que, la literatura como principal indicador de esta 
transición, es decir, completar sus estudios y entrar en el mercado de trabajo, dejando la casa de sus padres y ser capaz de seguir viviendo de forma independiente, así como casarse y tener sus hijos (Silva, 2008). De esta manera, los estudiantes universitarios pueden pasar por diferentes vías de transición universidad-empleo, teniendo en cuenta sus necesidades, habilidades, formación personal y académica, el apoyo familiar y las oportunidades. A lo largo de este trayecto en la búsqueda de un primer empleo 0 un trabajo en el ámbito de la formación, es el primer gran proyecto personal del individuo, con consecuencias en la salud y el bienestar psicológico (Sarriera, Schwarcz, \& Câmara, 1996; Ballester \& Fernández, 2015).

Hipótesis 5: Los individuos con graduación presentan un mayor desaliento ante la búsqueda de empleo e índices más bajos de salud mental.

Al completar la graduación, Ios individuos defienden una perspectiva de carrera, y cuando esta es afectada por el desempleo (Cruz, 2009, p.31), puede crear una amenaza potencial para las creencias personales de carrera. Este hecho puede proporcionar un desánimo frente a la búsqueda de empleo y por consiguiente síntomas psicológicos, como el estrés y la depresión.

Analizamos si los graduados presentan un mayor desánimo frente la busca de trabajo y por lo tanto un menor índice de la salud mental. Para responder a esta cuestión se analizaron las respuestas de los individuos al cuestionario JHS. La asociación de JHS con la calificación, muestra que relativamente al Grado, y al Máster, estos no presentan diferencias estadísticamente significativas, lo que indica que no es posible comprobar que los graduados tienen un mayor desánimo ante la búsqueda de empleo. A fin de comprender mejor esta cuestión, se utilizó la correlación entre la calificación, el JHS, los índices de BSI y los índices de MHI. La asociación de MHI con la calificación, muestra que para el índice global, éste presenta una débil asociación negativa, lo que indica que cuanto menor es la calificación, o sea, la muestra que se encuentra en el último año de la graduación, mayor es el nivel de la angustia que presenta, consecuentemente menor es el nivel del índice de salud mental. Según los resultados obtenidos, no fue posible comprobar de forma concluyente si los graduados presentan un menor índice de salud mental en comparación con los que se encuentran a asistir el máster. Sin embargo se encontró una débil asociación entre la graduación y un menor índice de salud mental, luego una mayor angustia psicológica. Este resultado va en la misma dirección de la Hipótesis 2, en el que los estudiantes universitarios que están en el último año de la graduación, presentan un mayor nivel de angustia psicológica y por lo tanto, una menor salud mental.

Las personas siempre han esperado que estudiando más tempo tendrían una garantía de empleo y, luego, después de los 16017 años estudiando de forma a obtener una graduación, tendrían una mayor oportunidad de desarrollar una carrera estable, con la posibilidad de ascenso, un salario justo, buenas condiciones de trabajo y realización profesional (Cruz, 2009). Sin embargo, en la actualidad, la construcción de una carrera por parte de los jóvenes titulados, es muy difícil, sobre todo la existencia de un mercado inestable, con cambios tecnológicos, reestructuraciones empresariales y precariedad, pero sobre todo por los altos niveles de desempleo, que afectan particularmente a los jóvenes a la salida de la enseñanza superior. Así, la situación de los individuos con grado, que perciben obtener un empleo futuro, pero que abogan un sentimiento de "vacío" y la impotencia frente a las dificultades en acceder al mercado laboral, tienen un menor nivel de bienestar psicológico y experimentan consecuencias negativas con respecto a la salud mental (Sarriera \& Verdin, 1996 cit. in Coelho \& Aquino, 2009, p.280; Ballester \& Fernández, 2015).

Por consiguiente, los individuos que completan el grado, tienden a experimentar altos niveles de depresión, ansiedad, estrés y angustia en general juntamente con baja autoestima y baja con- 
fianza (Cruz, 2009), proporcionando un mayor desánimo ante la búsqueda de empleo. Así, los futuros profesionales deben, allá de las competencias profesionales técnicos, desarrollar competencias personales y sociales, en la transición hacia el mercado laboral (Ballester \& Fernández, 2015). Estas competencias se centran principalmente en las capacidades de comunicación, organización y gestión del tiempo, trabajo en equipo y gestión de conflictos y el estrés. También la transición al mercado de trabajo es una de las etapas de la vida académica de cada estudiante que puede potenciar ansiedad y vulnerabilidad. En este contexto, el estudiante debe tener un conjunto de competencias específicas, tales como: las competencias de auto-regulación, pro-actividad y estrategias de coping frente a situaciones de conflicto y ansiedad.

Hipótesis 6: El desaliento frente a la demanda de empleo, siendo inversamente proporcional al índice de síntomas psicopatológicos.

Para responder a esta pregunta de investigación, se utilizó la muestra con todos los individuos $(n=302)$ con el fin de poder comparar el desaliento ante la búsqueda de empleo (JHS) y las dimensiones y la escala de los índices de BSI. Los resultados muestran una débil correlación negativa, principalmente entre JHS y la Depresión, entre el JHS y entre el JHS y el Índice de Síntomas Positivos. Relativamente a las correlaciones menos elevadas pudimos comprobar la asociación entre el Índice Total de Síntomas Positivos y JHS y entre la dimensión Ideación Paranoide y JHS. Es decir, cuanto menor el desaliento ante la búsqueda de empleo, mayor es la sintomatología psicopatológica presentada.

Así que, de acuerdo con 0' Brian (2002 cit. in Claudino \& Cordeiro, s/d, p.199) entendemos el desaliento ante la búsqueda de empleo, como una perturbación de adaptación, como algo que se refiere a las situaciones en la que el individuo desarrolla determinados síntomas psicológicos en respuesta a factores de estrés, o como una falta de adaptación superficial a situaciones difíciles a nuevos factores ambientales experimentados. Teniendo en cuenta al individuo como un ser holístico y único, es esencial tener en cuenta la totalidad del compromiso cultural, persona étnica y social para evaluar los mecanismos de adaptación, con el fin de concluir, si el comportamiento demostrado es inadecuado, aceptable 0 apropiado. Por lo tanto, los síntomas exhibidos por el individuo, no son más que una forma inconsciente de responder a un estrés particular. El individuo cuando expuesto a un factor estresante particular, puede sentir limitaciones 0 incapacidades relativamente a su funcionamiento social, ocupacional o académico. Esta reacción es generalmente relacionada con la necesidad de ajuste necesario del individuo en momentos de cambio (Claudino \& Cordeiro, s/d). El estrés interfiere de tal manera con los procesos cognitivos, en la que el individuo se siente incapaz de reunir la información que necesita para poder comprender, las relaciones causa-efecto y ser capaz de seleccionar la alternativa más adecuada para la toma de decisiones, quedando así incapaz de hacer frente a los graves problemas con los que tenga que confrontarse. Un individuo, cuando no consigue ajustar mecanismos eficaces de adaptación, manifiesta comportamientos depresivos y es incapaz de responder de forma adecuada a la situación de estrés como en el caso de encontrar un empleo.

Como se esperaba, se encontró una asociación inversamente proporcional y significativa, aunque débil, entre el desaliento ante la búsqueda de empleo y los síntomas psicopatológicos. Este resultado va en la dirección de Lynd-Stevenson (1997 cit. in Cruz, 2009, p.95), al referirse que el desaliento relacionado con el empleo, pueda medir, pero no reducir la relación entre la vulnerabilidad de los factores y la depresión, cuando las expectativas relacionadas con el empleo no se realizan, es decir, los individuos se desaniman, quedándose ante una situación de depresión considerando que nada puede revertir la situación. 
Hipótesis 7: El desaliento ante la búsqueda de empleo tiene influencia en la angustia y el bienestar psicológico.

Siendo la búsqueda de empleo una experiencia que influye en los acontecimientos de la vida de los sujetos de la muestra, estos pueden encontrarse con grandes niveles de desánimo si no consiguen sus objetivos. Así que, al tratar con estas situaciones, puede haber una grande posibilidad de quedarse depresivos o presentaren otros factores de vulnerabilidad durante este período. Por lo tanto, examinamos a través de una correlación, el desaliento ante la búsqueda de empleo y las dimensiones e índices de escala del MHI. Constatamos que en su totalidad, las correlaciones entre el JHS y las dimensiones y los índices del MHI tienen una correlación positiva, principalmente entre el JHS y el Pérdida del Control Emocional/Conductual, entre el JHS y el la Angustia psicológica y entre el JHS y el índice global del MHI. Relativamente a las correlaciones menos elevadas, podemos verificar la asociación entre el índice Depresión y JHS, y entre la dimensión Lazos emocionales y JHS.

Los resultados muestran así, que existen una asociación entre el desánimo ante la búsqueda de empleo y la angustia y el bienestar psicológico evaluado por el MHI, donde se percibe que cuanto mayor es el desaliento ante la búsqueda de empleo, mayor es la angustia y bienestar psicológico. Este resultado va también en dirección con la versión de Lynd-Stevenson (1997 cit. in Cruz, 2009, p.95), cuando se refiere que los individuos con grandes niveles de desánimo indican una ausencia de capacidad para hacer frente a situaciones traumáticas que ocurren en sus vidas y una gran posibilidad de deprimirse durante estos eventos traumáticos, mientras pequeños niveles de desaliento presentados por individuos indican una capacidad para hacer frente a estos eventos y una diminuta posibilidad de deprimirse durante la duración de estos acontecimientos, como en el caso de una situación de búsqueda de empleo. Así que, las evidencias encontradas frente al desánimo relacionado con el empleo, presupone que las expectativas relacionadas al empleo no se realicen, los individuos se desaniman y quedan ante una situación de depresión, considerando que nada va a pasar para que la situación se revierta.

El empleo tiene hoy una función integradora y complementar de la personalidad individual ya que se valora hasta el punto de convertirse en un papel central en la vida de los individuos, entonces, es de creer que el desempleo afectará de alguna manera el bienestar subjetivo de los individuos. Así, se cree que el desempleo tendrá un significado psicológico diferente para cada uno de los individuos, y consecuentemente, y manifestando sus actitudes y comportamientos. Es importante señalar que la situación de empleo entre los jóvenes tiene consecuencias negativas en relación con su salud. Al finalizar el grado, Ios individuos luchan por una perspectiva de carrera, y cuando esta es afectada por el desempleo (Cruz, 2009, p.31), puede crear una amenaza potencial para las creencias personales de carrera. Puede llevar a un proceso psicológico de luto, lo que se traduce en particular, en la destruición de los mitos y de las creencias vocacionales del individuo. El desempleo exige así más del individuo, viéndose en la necesidad de reorganizarse y reorientar su itinerario profesional de forma a desarrollar e implementar el concepto de sí. La transición y la integración de los recién licenciado en la vida laboral y búsqueda de empleo, requiere una (re)organización en la gestión de los recursos disponibles, una buena gestión de conflictos y de estrés en contextos muy exigentes, de modo a ser experimentada con éxito y bienestar.

La transición para el mercado laboral de los estudiantes universitarios, requiere un desarrollo de competencias y estrategias por ellos, con el objetivo de mejorar su desempeño social y la capacidad de gestión de situaciones de estrés con reflejos positivos en su calidad de vida y sentimiento de auto-eficacia. De esta forma se debe optimizar las estrategias de búsqueda de empleo a fin de promover una transición e integración con éxito en el mercado laboral (Ballester \& Fernández, 2015). 
Así, podemos decir que la búsqueda de un puesto de trabajo, puede proporcionar al individuo el reconocimiento de una discrepancia entre lo que aspira y lo que desea desempeñar (Cruz, 2009), lo que puede ser muy angustiante, pero al mismo tiempo, puede estimular la exploración de nuevos valores y la reformulación del concepto de sí mismo.

\section{CONSIDERACIONES FINALES}

El objetivo general de esta investigación pretende ser una contribución para promover la reflexión, el análisis y la comprensión de los aspectos psicológicos cuando los estudiantes salen de las Universidades intentando entrar en la vida profesional.

Los resultados encontrados en nuestro estudio son consistentes con las referencias teóricas abordadas en la revisión de la bibliografía. En conclusión, con respecto a la salud mental, podemos decir que los datos sugieren que no habrá diferencias significativas entre mujeres y hombres en la prevalencia general. Y que los problemas de salud mental empiezan en la edad universitaria típica, entre 18 y 24 años, aunque los estudiantes universitarios no tienen más problemas psiquiátricos que otros jóvenes no universitarios, éstos muestran un mayor nivel de Ansiedad, Depresión y Pérdida del Control Emocional/Conductual (dimensiones negativas del MHI), congruente con los resultados de nuestro estudio. Los licenciados desarrollan expectativas superiores de formación profesional, por lo que cuando una situación de no-integración profesional, estos son objeto de impactos psicosociales y menor salud mental. Es importante señalar que la situación de desempleo entre los jóvenes tiene consecuencias negativas en relación con su salud. Así, se puede mencionar que los jóvenes licenciados tienen un menor nivel de bienestar psicológico, debido a un sentimiento de "vacío" y la impotencia frente a las dificultades para acceder al mercado laboral. En consecuencia los individuos que completen el grado, tienden a experimentar elevados niveles de depresión, ansiedad, estrés y angustia en general, junto con baja confianza y baja autoestima dando lugar a un mayor desánimo ante la búsqueda de empleo.

Podemos concluir que los resultados de la investigación, muestran que existe una asociación entre el desánimo ante la búsqueda de empleo y los síntomas psicopatológicos evaluados por el BSI y que existe una asociación entre el desánimo ante la búsqueda de empleo, la angustia y el bienestar psicológico evaluado por el MHI.

En síntesis, nuestro estudio refuerza la importancia de la salud mental del joven universitario en busca de trabajo. En este sentido, es fundamental la comprensión del proceso de desarrollo en función de los vectores asociados a las diferentes etapas de crecimiento personal y académico, que pasa por la gestión de competencias y emociones, por la construcción de la autonomía y la identidad hasta la construcción de los valores y la integridad personal (Evans et al., 1998 cit. in PortaNova, 2009).

\section{REFERENCIAS BIBLIOGRÁFICAS}

Aguiar-Conraria, L.; Alexandre, F. \& Pinho, M. (2010). 0 euro e o crescimento da economia portuguesa: uma análise contrafactual. Núcleo de Investigação em Políticas Económicas da Universidade do Minho.

Alarcão, M. \& Sousa, L. (2007). Rede social pessoal: do conceito à avaliação. Psychologica, 44, pp.353-376.

Almeida, L. (2004). A juventude universitária e a nova sociabilidade: continuidade ou ruptura? VIII Congresso Luso-Afro-Brasileiro de Ciências Sociais. Centro de Estudos Sociais na Faculdade de Economia da Universidade de Coimbra.

Alves, N. (2007). Inserção profissional e formas identitárias: percursos dos licenciados da 
Universidade de Lisboa. Dissertação de Doutoramento da Universidade de Lisboa.

Amaro, F. (2004). A família portuguesa - tendências actuais. Cidade Solidária - Revista da SCML. Julho, pp.1-5.

Assis, A. \& Oliveira, A. (2011). University life and mental health: Customer demands for health and mental health of students at a brazilian university. Cadernos Brasileiros de Saúde Mental, 2(45), pp.159-177.

Ballester, S. \& Fernández, M. (2015). Valores de éxito y emprendimiento. International Journal of Developmental and Educational Psychology - INFAD, 2(1), pp.171-184.

Banco de Portugal. (2013). Projecções para a Economia Portuguesa: 2013-2014. Boletim Económico, pp. 39-46.

Barnes, J. \& Muller, L. (2000). Promoção da saúde mental: uma perspectiva desenvolvimental. Psychology, Health \& Medicine, 2(5). Setembro/Outubro, pp.17-27.

Barros, C. \& Oliveira, T. (2009). Mental health of unemployed workers. rPOT 9(1). Janeiro/Junho, pp.86-107.

Caleiras, J. (2011). Do desemprego à pobreza: trajectórias de empobrecimento, experiências vividas e políticas públicas. [Electronic Version]: Retirado de https://estudogeral.sib.uc.pt/bitstream/10316/16439/3/Tese\%20Doutoramento\%20Jorge\%20C aleiras.pdf, acedido em 14/05/2012.

Campos, A. \& Conceição, H. (2007). Mercado de trabalho, emprego e desemprego. Instituto Superior de Engenharia de Coimbra.

Canavarro, M. (1999). Inventário de Sintomas Psicopatológicos: BSI. In M. Simões, M. Gonçalves, \& L. Almeida (Eds.), Testes e provas psicológicas em Portugal (vol.II, pp.87-109). Braga: SHO/APPORT.

Canavarro, M. (2007). Inventário de Sintomas Psicopatológicos: Uma revisão crítica dos estudos realizados em Portugal. In M. Simões, C. Machado, M. Gonçalves, \& L. Almeida (Eds.), Avaliação psicológica: Instrumentos validados para a população Portuguesa (vol.III, pp.305331). Coimbra: Quarteto Editora.

Cardoso, R. \& Sampaio, H. (1994). Estudantes universitários e o trabalho. Revista Brasileira de Ciências Sociais, 26.

Carvalho, J. (2012). Os "novos mundos da inserção" dos/as diplomados/as do ISMT: Precariedade e individualização nas transições actuais para o mundo do trabalho. Dissertação de Mestrado em Gestão de Recursos Humanos e Comportamento Organizacional submetido ao Instituto Superior Miguel Torga: Coimbra.

Castelhano, L. (2006). A perda do emprego, suas implicações subjectivas e as consequências para o laço social: uma contribuição psicanalítica. Dissertação de Mestrado em Psicologia Social submetido à Pontifícia Universidade Católica de São Paulo.

Cerchiari, E.; Caetano, D. \& Faccenda, 0. (2005). Prevalência de transtornos mentais menores em estudantes universitários. Estudos de Psicologia, 10(3): pp.413-420.

Claudino, J. \& Cordeiro, R. (2006). Níveis de ansiedade e depressão nos alunos do curso de licenciatura em enfermagem: o caso particular dos alunos da Escola Superior de Saúde de Portalegre. [Electronic Version]: Millenium - Revista do ISPV, 32, pp.197-210. Retirado de http://www.ipv.pt/millenium/Millenium32/15.pdf, acedido em 11/07/2007.

Coelho, R. \& Aquino, C. (2009). Inserção Laboral, Juventude e Precarização. Psicologia Política, 9(18), pp.275-289.

Coelho, V. \& Ornelas, J. (2010). Os contributos do emprego apoiado para a integração das pessoas com doença mental. Análise Psicológica, 3(XXVIII), pp.465-478. 
Comissão Europeia (2001). Relatório "Os objectivos futuros concretos dos sistemas de educação e formação". Bruxelas: Comissão Europeia, Direcção Geral de Educação e Cultura.

Costa, E. (2004). Saúde mental, estratégias de coping e adaptação académica - uma investigação com estudantes universitários do segundo ano de Viseu. Dissertação de Mestrado em Psicologia da Saúde submetida ao Instituto Superior de Psicologia Aplicada. Lisboa.

Cruz, M. (2009). O Impacto do desemprego nos níveis da depressão, auto-estima e desânimoaprendido - Estudo comparativo numa amostra de empregados e desempregados. Dissertação de Mestrado em Psicologia do Trabalho e das Organizações submetida à Universidade Fernando Pessoa.

Derogatis, L. (1993). BSI: Brief Symptom Inventory. Administration, Scoring and Procedures. Manual. Minneapolis: National Computers Systems.

Dornelas, A.; Ministro, A.; Lopes, F.; Albuquerque, J.; Paixão, M. \& Santos, N. (2010). Emprego, contratação colectiva de trabalho e protecção da mobilidade profissional em Portugal. Estudo elaborado por solicitação da Ministra do Trabalho e da Solidariedade Social de Portugal.

Eurydice \& Eurostat (2003). Key data on education in Europe 2002. Bruxelas: Comissão Europeia.

Feitosa, F; Matos, M.; Del Prette, Z. \& Del Prette, A. (2005). Suporte social, nível socioeconómico e 0 ajustamento social e escolar de adolescentes portugueses. Temas em Psicologia, 13(2), pp.129-138.

Ferraz, M. \& Pereira, A. (2002). A dinâmica da personalidade e o Homesickness (saudades de casa) dos jovens estudantes universitários. Psicologia, Saúde \& Doenças, 3 (2), pp.149-164.

Freitas, M. (2007). Sobre a perda de ímpeto no processo de convergência da economia Portuguesa: uma abordagem dogmática. Notas Económicas da Faculdade de Economia - Universidade de Coimbra. Junho, 25, pp.27-41.

García, B. \& Torres, B. (2008). Acceso al mercado laboral de las tituladas superiores en España: empleabilidad y cualificaciónnfor. Madrid: Ministerio de Igualdad.

Gomes, C. (2003). Novo modelo de intervenção em contexto de transição para a vida activa. [Electronic Version]: Retirado de sigarra.up.pt/.../pt/publs_pesquisa.show_publ_file, acedido em 14/05/2012.

Gondim, S. (2002). Perfil profissional e mercado de trabalho: relação com a formação académica pela perspectiva de estudantes universitários. Estudos de Psicologia, 7(2), pp.299-309.

Gonçalves, J. (2009). Desenvolvimento profissional e carreira docente - fases da carreira, currículo e supervisão. Sísifo: Revista de Ciências da Educação, 8, pp.23-36.

GTAEDES (2010). Doença mental em estudantes universitários. II Seminário do GTAEDES. [Electronic Version]: Retirado de http://www.aminharadio.com/gtaedes/sites/default/files/artigo_aserra.pdf, acedido em 14/05/2012.

Guadalupe, S. (2008). A saúde mental e o apoio social na família do doente oncológico. Dissertação de Doutoramento em Saúde Mental submetida ao Instituto de Ciências Biomédicas de Abel Salazar, Universidade do Porto.

Instituto Nacional de Estatística. (2013). Desemprego em portugal. [Electronic Version]: Retirado de http://www.ine.pt/xportal/xmain?xpid=INE\&xpgid=ine_sem_lista\&xlang=pt, acedido em $14 / 05 / 2012$.

Jerónimo, M. (2010). O papel da acção social na igualdade de oportunidades de acesso e frequência de estudantes do ensino superior politécnico público português. Dissertação de Doutoramento em Didáctica e Organização Escolar, submetida à Faculdade de Educação da Universidade da Extremadura.

Joia, L. (2010). Profile lifestyle staff between students from a college. Revista Movimenta, 3(1), pp.16-23. 
Kilimnik, Z. (1998). Trabalhar em tempos de "Fim dos Empregos": Mudanças na trajectória de carreira de profissionais de recursos humanos. Psicologia Ciência e Profissão, 18(2), pp.34-45.

Kilimnik, Z.; Castilho, I. \& Sant'Anna, A. (2006). Carreiras em transformação e seus paradoxais reflexos nos indivíduos: metáforas de carreira e de competências. Comportamento Organizacional e Gestão, 12(2), pp.257-280.

Königstedt, M. (2008). Educação e carreira - estudo de avaliação da eficácia de uma intervenção psicológica. Dissertação de Mestrado em Psicologia - Especialização em Psicologia Escolar submetida a Universidade do Minho - Instituto de Educação e Psicologia.

Lynd-Stevenson, R. (1996). A testo $f$ the hopelessness theory of depression inemployed young adults. British Journal of Clinical Psychology, 35, pp.117-132.

Lynd-Stevenson, R. (1997). Generalized and event-specific hopelessness: salvaging the mediation hypothesis of the hopelessness theory. British Journal of Clinical Psychology, 36, pp.73-83.

Maroco, J. (2007). Análise estatística com utilização do SPSS (3ª ed.). Lisboa: Edições Sílabo.

Marques, R. (2003). Saúde mental para e pelo trabalho. Trabalho apresentado na disciplina de Crítica ao Direito do Trabalho, do curso de Pós-Graduação em Direito. Universidade Federal do Paraná.

Marques, V. (2011). Influência do suporte social e dos estilos de coping na percepção de bem-estar subjectivo em doentes mentais crónicos. Dissertação de Mestrado em Psicologia Clínica submetida ao Instituto Superior Miguel Torga.

Martins, M. (2002). Qualidade de vida e capacidade para o trabalho dos profissionais em enfermagem no trabalho em turnos. Dissertação apresentada ao programa de Pós-Graduação em Engenharia de Produção da Universidade Federal de Santa Catarina como requisito parcial para obtenção do grau de Mestre em Engenharia de Produção - Área de Ergonomia.

Maslach, C. \& Jackson, S. (1986). Maslach Burnout Inventory. Second Edition. Palo Alto, CA: Consulting Psychologists Press.

Matos, A. (2004). Saúde mental. Lisboa: Climepsi Editores.

ME-DGES. (1999). O ensino superior em portugal. Lisboa: Direcção-Geral do Ensino Superior, Ministério da Educação.

Medina-Mesa, Y.; Martínez-García, C.; Morales-Sánchez, L. \& Gil-Olarte, P. (2018). Las actitudes del alumnado universitário frente a las personas com trastorno mental. International Journal of Developmental and Educational Psychology - INFAD, 1(2), pp.209-218.

Meireles, D. \& Spode, C. (2010). Workshops for work placement preparation: supporting young job seekers. Revista Conhecimento Online. Março, 2(1). [Electronic Version]: Retirado de www.feevale.br/revistaconhecimentoonline, acedido em 14/05/2012.

Melo, S. \& Borges, L. (2007). A Transição da universidade ao mercado de trabalho na ótica do jovem. Psicologia Ciência e Profissão, 27 (3), pp.376-395.

Melvin, P. (1978). In: 0 correio de unesco. Janeiro, 7(1), pp.34. [Electronic Version]: Retirado de http://www.eumed.net/cursecon/libreria/2004/lgs-mem/9.htm, acedido em 14/05/2012.

Mercuri, E.; Polydoro, S.; Santos, A.; Prette, A.; Soares, A.; Fior, C.; Pereira, E.; Pachane, G.; Bariani, I.; Almeida, L.; Primi, R.; Zenorine, R. \& Prette, Z. (2004). Estudante universitário: características e experiências de formação. Colecção Psicologia e Educação Superior. São Paulo: Cabral Editora e Livraria Universitária.

Ministério da Educação e Ciência. (2013). Fundação para a ciência e tecnologia. [Electronic Version]: Retirado de http://www.fct.pt/apoios/bolsas/valores.phtml.pt, acedido em 14/05/2012.

Monteiro, I. (2009). O contributo das experiências familiares, vinculação e apoio social para a depressão no adulto. Dissertação de Doutoramento em Psicologia Clínica da Universidade do 
Minho - Instituto de Educação e Psicologia.

Motta, P. (2002). Ansiedade e medo no trabalho: a percepção do risco nas decisões administrativas. VII Congreso Internacional del CLAD sobre la Reforma del Estado y de la Administración Pública, Lisboa, pp.8-11.

Myers, J. \& Well, A. (2003). Research design and statistical analysis. New Jersey: Lawrence Erlbaum Associates.

Narciso, I. \& Ribeiro, M. (2009). Olhares sobre a conjugalidade. Lisboa: Coisa de Ler.

Neiva, K. (1996). Fim dos estudos universitários: efeitos das dificuldades do mercado de trabalho na representação do futuro profissional e no estabelecimento de projectos pós-universitários dos estudantes. Psicologia USP, 7(1/2), pp.203-224.

Neves, J. (2008). Princípios de economia política. Lisboa: Editorial Verbo.

Neves, M. \& Dalgalarrondo, P. (2007). Self-referred mental disorders in university students. Jornal Brasileiro de Psiquiatria, 56(4), pp.237-244.

Nogueira, D.; Pereira, L. \& Ferreira, M. (2007). Desenvolvimento de carreira e saúde mental. Trabalho realizado no âmbito da disciplina de Orientação Escolar e Profissional I, do 4ํano da licenciatura de Psicologia da Universidade da Beira Interior.

Observatório da Ciência e do Ensino Superior (2004). O sistema do ensino superior em Portugal (1993- 2003). Lisboa: Ministério da Ciência, Inovação e Ensino Superior.

Oliveira, D.; Fischer, F.; Teixeira, M. \& Gomes, A. (2005). Adolescência, trabalho e estudo: análise comparativa das representações sociais de adolescentes trabalhadores e não trabalhadores. Revista de Enfermagem - UERJ, 13, pp.229-239.

Osuna, F. (2003). Procesos de transición al nercado de trabajo: estrés y prácticas en empresa de la Universidad de Granada. Dissertação de Doutoramento em Aplicações e Métodos em Psicologia Social submetida à Universidade de Granada.

Pacheco, J. (2003). Políticas educativas para o ensino superior na União Europeia: um olhar do lado português. Educação Social, 24(82), pp.17-36.

Pedroso, P.; Ferreira, A.; Dornelas, A.; Estanque, E.; Centeno, M.; Novo, A. \& Henriques, M. (2005). Relatório final sobre o acesso ao emprego e mercado de trabalho - formulação de políticas públicas no horizonte de 2013. Faculdade de Economia da Universidade de Coimbra.

Pereira, A. (2006). Stresse e doenças: contributos da psicologia da saúde na última década. Perspectivas em Psicologia da Saúde, pp.145-167. Coimbra: Quarteto Editora.

Pereira, A. (2009). Análise de depressão e ansiedade nos alunos do ensino superior: comparação com um estudo do curso de radiologia. Trabalho realizado para a Escola Superior de Saúde Dr. Lopes Dias. Instituto Politécnico de Castelo Branco.

Pereira, M. (2012). Vulnerabilidade ao stress e mecanismos de coping na elaboração da dissertação dos alunos do segundo ciclo de psicologia de uma instituição de ensino superior privado. Dissertação de Mestrado em Psicologia Clínica, Ramo Psicoterapia e Psicologia Clínica submetido ao Instituto Superior Miguel Torga.

Pereira, A.; Motta, E.; Vaz, A.; Pinto, C; Bernardino, 0.; Melo, A.; Ferreira, J.; Rodrigues, M.; Medeiros, A. \& Lopes, P. (2006). Sucesso e desenvolvimento psicológico no ensino superior: estratégias de intervenção. Análise Psicológica, 1(XXIV), pp.51-59.

Pinheiro, M. \& Ferreira, J. (2001). Avaliação do suporte social em contexto de ensino superior. Faculdade de Psicologia e de Ciências da Educação da Universidade de Coimbra. Comunicação apresentada no V Seminário de Investigação e Intervenção Psicológica no Ensino Superior. Viana do Castelo.

Pinheiro, L. \& Monteiro, J. (2007). Refletindo sobre desemprego e agravos à saúde mental. 
Cadernos de Psicologia Social do Trabalho, 10(2), pp.35-45.

Piteira, C. (2012). 0 impacto da crise económica na saúde. [Electronic Version]: Retirado de http://opaisquetemos.wordpress.com/2012/11/, acedido em 14/05/2012.

Polo, A.; Hernández, J. \& Poza, C. (1996). Evaluación del estrés académico en estudiantes universitários. Universidad Autónoma de Madrid. Ansiedad y Estrés, 2, pp.159-172.

Porta-Nova, R. (2009). Adaptabilidade, competências pessoais e bem-estar psicológico de jovens do ensino superior na área das ciências da saúde. Dissertação de Doutoramento em Saúde Mental submetida ao Instituto de Ciências Biomédicas de Abel Salazar da Universidade do Porto.

Portal da Saúde. (2012). Programa Nacional para a Saúde Mental. [Electronic Version]: Retirado de http://pns.dgs.pt/), acedido em 14/05/2012.

Precioso, J. (2004). Educação para a saúde na universidade: um estudo realizado em alunos da Universidade do Minho. Revista Electrónica de Ensenanza de las Ciencias, 3(2).

Rabasquinho, C. \& Pereira, H. (2007). Género e saúde mental: uma abordagem epidemiológica. Análise Psicológica, 3(XXV), pp.439-454.

Ramos, S. \& Carvalho, A. (2007). Nível de stress e estratégias de coping dos estudantes do 1ํano do ensino universitário de Coimbra. Portal dos Psicólogos. [Electronic Version]: Retirado de www.psicologia.com.pt, acedido em 14/05/2012.

Rebelo, G. (2010). Trabalho e emprego: actualidade e prospectiva. Lisboa: Edições Sílabo.

Reis, L.; Nogueira, L.; Rocha, M. \& Marangoni, 0. (2002). Qualidade de vida no trabalho. Trabalho apresentado ao 2.ำ período do curso de Administração da Pontifícia Universidade Católica de Minas Gerais - Campus Poços de Caldas.

Relatório Emprego. (2012). A evolução recente do desemprego. Relatório elaborado pelo Ministério das Finanças, Ministério da Economia e Emprego, Ministério da Solidariedade e Segurança Social de Portugal.

Relvas, A. (1996). O ciclo vital da família - perspectiva sistémica. Porto: Edições Afrontamento.

Relvas, A. \& Alarcão, M. (2002). Novas formas de família. Coimbra: Quarteto.

Ribeiro, J. (1999). Investigação e avaliação em psicologia e saúde. Lisboa: Climepsi Editores.

Ribeiro, J. (2001). Mental health inventory: um estudo de adaptação à população portuguesa. Psicologia, Saúde \& Doenças, 2(1), pp.77-99.

Rocha, M. (2009). Formação e transição para o mundo do trabalho através das tecnologias da informação e comunicação: as novas desigualdades de género. Actas do X Congresso Internacional Galego-Português de Psicopedagogia. Braga: Universidade do Minho.

Rodrigues, V. \& Madeira, M. (2009). Suporte social e saúde mental: revisão da literatura. Revista da Faculdade de Ciências da Saúde, 6, pp.390-399. Porto: Edições Universidade Fernando Pessoa.

Sarriera, J.; Schwarcz, C. \& Câmara, S. (1996). Juventude, ocupação e saúde. Trabalho desenvolvido pelo Grupo de Pesquisa em Psicologia Comunitária - PUCRS e Financiamento do CNPq e FAPERGS, pp.61-78. Porto Alegre: Instituto de Psicologia.

Seco, G.; Filipe, L.; Pereira, P. \& Alves, S. (2009). Transição para o mercado de trabalho: competências pessoais e sociais. Actas do X Congresso Internacional Galego-Português de Psicopedagogia, pp.1638-1653. Braga: Universidade do Minho.

Seco, G.; Pereira, I.; Dias, I.; Casimiro, M. \& Custódio, S. (2005). Para uma abordagem psicológica da transição do ensino secundário para 0 ensino superior: pontes e alçapões. Psicologia $e$ Educação, IV(1), pp.7-21.

Silva, A. (2005). Formação, trabalho e aprendizagem ao longo da vida. Universidade do Minho Instituto de Educação e Psicologia, pp.2071-2086.

Silva, A. (2008). A construção de carreira no ensino superior. Dissertação de Doutoramento em 
Psicologia Vocacional. Universidade do Minho - Instituto de Educação e Psicologia.

Silva, E. (1992). A inter-relação trabalho-saúde mental: um estudo de caso. Revista de Administração de Empresas, 32(4), pp.70-90.

Silva, M. (2004). Inserção profissional e condição social: trajectórias de jovens graduados no mercado de trabalho. Dissertação de Doutoramento apresentao ao Programa de Pós-Graduação em Educação da Universidade Federal de Santa Catarina.

Silva, P. (2008). Competências transversais dos licenciados e sua integração no mercado de trabaIho. Dissertação de Mestrado em Gestão de Recursos Humanos submetido à Universidade do Minho - Escola de Economia e Gestão.

Silveira, C.; Norton, A.; Brandão, I. \& Roma-Torres, A. (2011). Saúde mental em estudantes do ensino superior: experiência da consulta de psiquiatria do Centro Hospitalar São João. Acta Medica Portuguesa, 24(S2), pp.247-256.

Sluzki, C. (2007). Família e redes. In, Fernandes, L. \& Santos, M. (Coord.) Terapia familiar, redes e poética social. Lisboa: Climepsi Editores.

Soares, S. (2000). Desemprego e impacto psicossocial: estudo exploratório dos efeitos da situação profissional ao nível da auto-estima, desânimo aprendido face ao emprego e locus de controlo. Monografia apresentada à Universidade Fernando Pessoa.

Souza, M.; Daher, A. \& Baptista, M. (2010). Relação entre suporte familiar, saúde mental e comportamentos de risco em estudantes universitários. Acta Colombiana de Psicologia, 13(1), pp.143154.

Tavares, C. (2007). Políticas microeconómicas para Portugal - fundamentos, histórias e factos da reforma económica 2002-2004. Porto: Fubu Editores.

Tomé, T. (2012). Depressão e coping: estudo comparativo entre pessoas com deficiência mental e população em geral. Dissertação de Mestrado em Psicologia Clínica, Ramo Psicoterapia e Psicologia Clínica submetida ao Instituto Superior Miguel Torga.

Torres, L. (2008). Educação e trabalho: dinâmica da relação entre perfis de formação e perfis profissionais no campo dos recursos humanos. In Actas do VI Congresso Português de Sociologia - Mundos Sociais: Saberes e Práticas, Lisboa: Associação Portuguesa de Sociologia, pp.277293.

Valério, A.; Matos, L. \& Marques, M. (2006). Transição da escola para a vida adulta: uma experiência de aprendizagem integrada. Direcção Geral da Inovação e do Desenvolvimento Curricular, pp.1-10.

Vizzaccaro-Amaral, A. (2007). Dimensões psicossociais do trabalho precarizado e do desemprego em meio à actual crise socioeconómica mundial. Dissertação de Doutoramento em Ciências Sociais, Programa de Pós-Graduação em Ciências Sociais, Faculdade de Filosofia e Ciências de Marília-SP, Universidade Estadual Paulista.

Wickert, L. (2006). Desemprego e juventude: jovens em busca do primeiro emprego. Psicologia, Ciência e Profissão, 26(2), pp.258-269.

Zanello, V. \& Silva, R. (2012). Saúde mental, género e violência estrutural. Revista Bioética, 20(2), pp.267-279.

Zanini, D.; Verolla-Moura, A. \& Queiroz, I. (2009). Apoio social: aspectos da validade de constructo em estudantes universitários. Psicologia em Estudo, 14(1), pp.195-202. 